\title{
Laboratorio di Italiano come L2 per alunni di recente immigrazione
}

Federica Gaetano

\section{(2) OpenEdition \\ 1 Journals}

Edizione digitale

URL: http://journals.openedition.org/esp/3803

DOI: 10.4000/esp.3803

ISSN: 2532-0319

Editore

Centre d'Information sur l'Éducation Bilingue et Plurilingue

\section{Edizione cartacea}

Data di pubblicazione: 1 juin 2019

Paginazione: 37-48

ISSN: 1127-266X

Notizia bibliografica digitale

Federica Gaetano, «Laboratorio di Italiano come L2 per alunni di recente immigrazione», Éducation et sociétés plurilingues [Online], 46 | 2019, Messo online il 01 février 2021, consultato il 02 mars 2021. URL: http://journals.openedition.org/esp/3803 ; DOI: https://doi.org/10.4000/esp.3803

Questo documento è stato generato automaticamente il 2 mars 2021.

(c) CIEBP 


\title{
Laboratorio di Italiano come L2 per alunni di recente immigrazione
}

\author{
Federica Gaetano
}

\section{Inquadramento del laboratorio in un contesto ben più complesso}

\section{Qualche dato sugli studenti stranieri in Italia}

1 Prima di entrare nel vivo del racconto di questa esperienza è opportuno gettare uno sguardo ai dati di contesto che permettono di offrire qualche informazione in più sul fenomeno degli alunni stranieri e di configurare questo circoscritto laboratorio didattico in un quadro più ampio rispetto alla scuola dove si è realizzato. Come fonte sono stati esaminati i dati che afferiscono all'ultima elaborazione dell'Ufficio di Statistica del MIUR che si attestano all'anno scolastico 2016 - 2017.

2 In questi anni l'Italia a livello demografico ha come elemento principale, che caratterizza con la sua dinamicità il panorama scolastico, un costante calo degli studenti italiani bilanciato dall'altrettanto finora costante crescita degli studenti di origine migratoria. Per offrire qualche dato nel quinquennio 2011/2012- 2015/2016 gli studenti italiani sono diminuiti di 193.000 unità, passando da 8.205.000 a 8.012.000 $(-2,3 \%)$, mentre gli studenti stranieri sono aumentati di 59.000 unità $(+7,8 \%)$ passando da 756.000 a 815.000 unità. Il bilancio dei due movimenti di segno opposto è un decremento di 133.000 unità circa $(-1,5 \%)$ della popolazione scolastica complessiva che passa da 8.960.000 a 8.827.000 studenti.

3 Nell'A.S. 2004/2005 gli studenti stranieri ammontavano a circa 371.000 unità, a 630.000 nel 2008/2009, per arrivare agli attuali 826.000, pari al 9,4\% del totale.

4 Il fenomeno non è distribuito in modo uniforme sul territorio italiano: basti dire che da sole le prime dieci province con il maggior numero di studenti stranieri assorbono il $41,3 \%$ del totale. La prima in graduatoria è la provincia di Milano con circa 85.100 
studenti, seguita da Roma, dove è stato realizzato il laboratorio di cui parleremo in seguito, con circa 62.100 .

5 Rispetto ai dati forniti sulla popolazione studentesca straniera è essenziale trovare delle chiavi di lettura e la prima, anche in considerazione della competenza linguistica in italiano, è che la percentuale degli studenti stranieri nati in Italia sul totale degli studenti stranieri, evidenzia che la maggioranza è costituita dagli alunni stranieri di seconda generazione. Rispetto agli studenti con cittadinanza non italiana la quota dei nati in Italia si avvicina infatti al $61 \%$, motivo per il quale sempre più spesso ci si riferisce a questi studenti con la definizione di "studenti con background migratorio".

Come insegnante è questo un dato evidente, riscontrato nella composizione di tutte le classi. Quello che invece va attentamente analizzato è il tipo di supporto e potenziamento linguistico di cui questi alunni hanno bisogno, elemento purtroppo spesso sottovalutato o addirittura non considerato, ma richiede invece un'attenta previsione degli interventi necessari da mettere in campo, sia con politiche adeguate che con azioni specifiche nelle singole scuole rispetto ai fabbisogni linguistici reali rilevati.

7 Se infatti l'obiettivo formativo è che gli studenti stranieri colgano le stesse opportunità dei loro coetanei italiani, non bisogna dare per scontato che l'appartenere ad una seconda generazione possa garantire loro una competenza linguistica sufficiente per poter vivere efficacemente l'esperienza scolastica. Basti a questo proposito rinviare ai dati di per sé allarmanti, che rilevano come in base ai rilevamenti pertinenti all'A. S. 2015/2016 il ritardo scolastico degli studenti sia aumentato con il crescere dell'età. A 14 anni, corrispondenti alla frequenza della prima classe di secondaria superiore, la percentuale degli studenti stranieri con percorso di studio regolare si ferma al 52\% mentre il $46 \%$ frequenta ancora una classe di scuola secondaria di I grado: di questi il $34,8 \%$ è in ritardo di un anno, il 9,5\% di due e l'1,7\% di tre anni. All'età di 18 anni la percentuale di studenti regolari è scesa al 30,4\% contro il 69,6\% in ritardo.

8 E se è vero che siamo purtroppo in Italia di fronte ad una grave crisi del sistema scolastico in generale, l'osservazione di questi dati alla luce del confronto tra studenti italiani e studenti stranieri evidenzia comunque che le distanze rimangono notevoli. A livello nazionale, gli studenti italiani in ritardo nella frequenza scolastica sono circa il $10,5 \%$, contro il $32,9 \%$ degli studenti stranieri. Nei singoli ordini di scuola, la distanza a sfavore degli studenti stranieri nella percentuale dei ritardi è di 11 punti percentuali nella scuola primaria $(1,8 \%$ contro $13,2 \%)$, di 29 punti percentuali nella secondaria di I grado $(6,6 \%$ contro $35,4 \%)$ e di ben 59 punti percentuali nella secondaria di II grado. Questo dato va correlato anche con il tasso di scolarità che considerando solo il momento successivo al termine della scuola dell'obbligo riferito agli alunni di 17 e 18 anni di età (ultimo biennio di secondaria II grado) diminuisce fino al $64,8 \%$ per gli studenti stranieri rispetto all' $80,9 \%$ degli studenti italiani.

9 Altro problema da considerare nell'inserimento degli alunni stranieri soprattutto in ambito metropolitano, prima di addentrarci in uno specifico contesto scolastico, sono gli istituti con alta concentrazione di studenti stranieri che rischiano la "ghettizzazione" da parte della popolazione nativa. Sul tema della distribuzione degli alunni nelle scuole e nelle classi il MIUR ha dato delle istruzioni con la CM 2/2010, stabilendo che il numero degli alunni con cittadinanza non italiana presenti in ciascuna classe non possa superare di norma il $30 \%$ del totale degli iscritti. 
10 Scendendo più nello specifico al target su cui si è concentrato l'intervento didattico realizzato, bisogna distinguere, nell'ambito degli studenti con cittadinanza non italiana, un altro sottogruppo specifico di cui è importante registrare la dinamica: si tratta dei neoarrivati, o meglio dagli studenti che entrano per la prima volta nel sistema scolastico italiano, praticamente gli unici a cui è ovviamente riconosciuto, vista la mancanza di conoscenza della lingua italiana, uno status di evidente svantaggio linguistico spesso sofferto, in assenza di interventi specifici, dagli insegnanti che si trovano in classe alunni che per molti mesi non sono in grado di seguire e interagire nella pratica didattica. La situazione di emergenza di dover imparare l'italiano è stata finora gestita dalle scuole grazie alla buona volontà di insegnanti e dirigenti che hanno risposto in alcuni casi al bisogno linguistico indipendentemente dal possesso di adeguate competenze glottodidattiche, con risultati quindi che andrebbero approfonditi anche nei casi positivi in cui sia stato previsto un supporto linguistico. In riferimento a ciò la normativa 107/2005 ha legiferato l'introduzione di una nuova classe di insegnamento (la A23) relativa all'insegnamento di lingua italiana per discenti di lingua straniera previsto per la scuola secondaria di primo e secondo grado, con l'inspiegabile esclusione della scuola primaria, ma tuttora si è in attesa di un concorso che era programmato per il 2018 e si attendono nuove indicazioni per la sua prossima pubblicazione.

11 Tornando agli alunni entrati per la prima volta nel sistema scolastico italiano, osserviamo dai dati come siano in costante decrescita negli ultimi cinque anni. Per quanto riguarda il Lazio e la scuola primaria si attestano sul 3,1\% rispetto al totale degli alunni stranieri.

Da considerare inoltre che l'altro ambito educativo in cui la scolarità degli studenti con cittadinanza non italiana è nettamente inferiore a quella degli italiani, riguarda la scuola dell'infanzia. Tra i 3 e i 5 anni i bambini con cittadinanza non italiana presenti nelle scuole rappresentano il 77\% dei bambini con cittadinanza non italiana residenti in Italia, mentre il dato raggiunge il $96 \%$ per i bambini italiani. Ciò evidenzia come alcuni bambini, pur essendo presenti in Italia, non frequentino però la scuola e accedano direttamente alla scuola primaria senza aver potuto cogliere prima l'opportunità di un apprendimento ludico e naturale della lingua italiana insieme ai loro coetanei italiani.

13 Per concludere l'inquadramento, la scuola primaria rappresenta l'ordine di scuola con il maggior numero di presenze rispetto al totale della popolazione scolastica straniera. La scuola elementare rimane infatti il settore che assorbe il maggior numero di studenti con cittadinanza non italiana. Nell'A.S. 2016/2017 ha registrato l'aumento più cospicuo di studenti, pari a circa 4.800 unità, costituendo il 10,8\% del totale degli alunni di questo ordine di scuola.

14 Gli studenti sui quali si è concentrata la nostra limitata esperienza didattica appartengono alle prime undici comunità stranieri presenti in Italia: Egitto, Filippine, Bangladesh e Tunisia.

\section{Il laboratorio di lingua italiana come L2: storia di un intervento didattico}

15 Come insegnanti sentiamo forte la necessità che i nostri interventi siano sostenuti e definiti entro una linea d'azione comune e condivisa, illuminata da riferimenti 
scientifici e normativi. In assenza di ciò la scuola primaria ha comunque realizzato nel corso degli ultimi venticinque anni moltissime esperienze volte all'insegnamento dell'italiano come seconda lingua per rispondere alle tante situazioni di emergenza. Quella qui proposta è una di esse.

L'intervento svolto nasceva dall'urgenza di offrire un sostegno linguistico ad alunni di recente immigrazione avendo ben presente i dati di contesto e le diverse problematiche che esprimevano rispetto agli alunni stranieri nati in Italia.

17 L'esperienza didattica, realizzata grazie all'iniziativa della Dirigente scolastica ${ }^{1}$, si è svolta nell'anno scolastico 2017-2018 presso l'Istituto Comprensivo N. M. Nicolai, localizzato nella periferia nord-est di Roma in una zona limitrofa al quartiere di San Basilio, caratterizzato da una forte presenza di stranieri e da un marcato disagio sociale.

Il laboratorio, alla sua prima edizione, si è costituito sin da subito come luogo privilegiato di accoglienza, ascolto ed espressione dei bisogni dei bambini stranieri rispetto alla loro competenza linguistica in un momento particolare della loro biografia: quello del loro ingresso in una scuola, situata in un ambito urbano vasto e problematico.

Il percorso ha richiesto diverse fasi. Dapprima vi è stata l'individuazione dei fabbisogni linguistici all'interno della scuola attraverso la richiesta a tutte le insegnanti sulla presenza di bambini stranieri arrivati da poco in Italia o con marcate difficoltà di comprensione/produzione dell'italiano. Tale richiesta in alcuni casi si è incontrata con l'espressione dell'estremo disagio da parte delle insegnanti per non riuscire a scambiare, a volte, con i bambini stranieri anche semplici messaggi legati alla quotidianità. Tali limiti avevano generato delle incomprensioni, con piccole conseguenze negative che ostacolavano ancor di più un sereno inserimento degli alunni nel nuovo contesto. In queste situazioni anche la comunicazione con le famiglie emergeva come problematica, non potendo assumere i bambini il ruolo dei mediatori linguistici tra i genitori e la scuola con il risultato di un mancato o limitato coinvolgimento dei genitori in alcuni momenti di condivisione di compiti tra scuola e famiglia.

In seguito sono stati individuati quattro bambini (tre di sei anni in prima e una di otto in terza) ed è stato successivamente richiesto alle loro famiglie il permesso per partecipare al laboratorio. L'inizio del laboratorio è stato a novembre, dopo un mese e mezzo dall'inizio del calendario scolastico.

21 Vale la pena ricordare le modalità di tale richiesta: da una parte la scuola era consapevole di offrire un offerta formativa in più, dall'altra si ravvisava la necessità di una forte chiarezza nei confronti dei bambini e delle loro famiglie nel presentare la proposta. Il tutto è stato facilitato dalle loro insegnanti di classe, che hanno assunto un ruolo centrale di mediazione in ogni passaggio come figure di riferimento verso le quali era già avvenuto un affidamento. Vi è stata quindi la presentazione dell'insegnante del laboratorio da parte dell'insegnante di classe e una successiva comunicazione diretta in inglese per i genitori del Bangladesh e delle Filippine e in un italiano semplificato ed essenziale per i genitori tunisini ed egiziani. Da parte loro vi è stato un unanime ed entusiasta consenso, con la richiesta in due casi su quattro di una eventuale attivazione di corsi di italiano per adulti. Sono state dunque alte, da parte dei genitori, le 
aspettative rispetto all'istruzione e la fiducia dimostrata alle insegnanti, atteggiamenti che come docenti riscontriamo ormai come sempre più rari nei genitori italiani.

È stato quindi spiegato ai bambini che avrebbero frequentato per due ore a settimana durante l'orario scolastico un laboratorio dove imparare l'italiano. L'insegnante del laboratorio prima degli incontri si recava personalmente nelle quattro classi frequentate dai bambini per prenderli, utilizzando anche questo momento di raccolta dei bambini come attività utile per far orientare i bambini nel nuovo spazio, favorire una loro presa di parola e per brevi comunicazioni di raccordo su quanto di significativo ci potesse essere tra insegnante del laboratorio e insegnante di classe, creando piano piano una dimensione di serenità nella quale operare un passaggio e un gioioso distacco dalla loro classe e di costituzione di un nuovo spazio e gruppo di apprendimento.

Particolare cura e attenzione è stata dedicata da parte di tutti gli educatori coinvolti in questo progetto affinché rientrasse nella routine scolastica e non come allontanamento coatto dalla classe, evitando di creare la percezione di una situazione scolastica ghettizzante tra neoparlanti dell'italiano, ma che piuttosto fosse percepita come offerta della scuola a bisogni individuali di crescita rispetto a competenze specifiche, con scambi continui tra ciò che avveniva nel laboratorio e ciò che si stava facendo in classe.

Particolarmente attrattivo è stato il luogo dove si è svolto il laboratorio: un'aula dotata di uno spazio d'apprendimento molto accogliente con disposizione ad isola dei tavoli, scaffali alle pareti con giochi e libreria, lavagna affissa e uno stereo utilizzato per l'ascolto di dialoghi o canzoni; un altro spazio è stato quello della ludoteca, più adatta ad ospitare alcuni giochi di movimento.

Per analizzare il livello di partenza degli alunni nel corso delle prime lezioni sono stati somministrati dei test di ingresso: la prima batteria riguardava la discriminazione tra due figure dell'immagine corrispondente alla parola nominata in un ambito lessicale di base; la successiva somministrazione riguardava la discriminazione tra due frasi molto semplici di descrizione della figura corrispondente. Sempre sul piano dell'oralità, avendo tre di questi quattro alunni capacità grafiche ancora molto limitate, la successiva prova era riferita all'esecuzione di comandi verbali di semplici azioni.

Per approfondire l'aspetto fonatorio e la produzione orale è stata avanzata la richiesta della ripetizione di semplici parole e frasi. Sono poi state proposte altre attività rispetto alla competenza grafica, come quella di copiare una semplice frase scritta alla lavagna.

Il test di ingresso si è concluso con una breve intervista fatta al bambino sul luogo di origine e le prime impressioni sulla nuova realtà.

Le reazioni dei bambini sono state diverse ed è qui necessario aprire una parentesi per delineare anche se brevemente, i diversi profili dei discenti:

la bambina tunisina ha dimostrato fin da subito, nonostante per lei l'ingresso a scuola fosse avvenuto solo a metà ottobre, grande motivazione e impegno, senso di intuito e spiccate capacità che la portavano a risolvere diverse situazioni pur senza conoscere il significato di molti vocaboli. È apparsa molto vivace, allegra e propensa a stabilire contatti positivi con gli altri, rispondendo con manifesta consapevolezza alle domande dell'insegnante sull'arrivo in Italia, il nome della città e del paese di origine.

30 Il bambino egiziano, l'unico ad aver frequentato anche qualche mese di scuola dell'infanzia in Italia, ha dimostrato invece di avere difficoltà nella produzione di semplici parole o frasi, ha comunque espresso impegno verso le attività proposte ma 
appariva insicuro e chiedeva di essere guidato nell'esecuzione delle consegne ritenendole a priori troppo complicate per lui. Alla domanda "Da dove vieni?" ha risposto: "Io tanti Egitto". È apparso subito molto estroverso, esuberante, capace di instaurare relazioni positive con l'intenzione di farsi benvolere dagli altri bambini. Pur essendo in Italia da più tempo rispetto agli altri, si esprimeva però in modo confuso, con frasi tronche prive di concordanza, vocaboli imprecisi e dimostrando evidenti difficoltà nell'esprimere una semplice frase completa. La sua comprensione era sicuramente superiore a quella degli altri alunni ma non utilizzava il bagaglio lessicale di cui disponeva e manifestava grande disorientamento.

31 La bambina del Bangladesh, l'unica di otto anni, non discriminava tra due parole o frasi molto semplici la figura corrispondente ed ha eseguito solo la metà dei comandi verbali in modo esatto. Ha manifestato fin da subito di tenerci molto a svolgere il lavoro con cura e attenzione e aveva grande padronanza della tecnica della lettura ma non coglieva il significato delle parole lette. Alla domanda sul paese di origine non ha risposto, ma alla domanda formulata in inglese ha risposto correttamente in questa lingua. La bambina è apparsa fin dalla prima osservazione molto timida e riservata, non si esprimeva affatto e sembrava non capire il significato di parole molto semplici e consegne elementari non potendo così accedere alla possibilità di uno scambio comunicativo anche a livello non verbale. Scriveva sotto dettatura e leggeva senza la comprensione delle parole ma con una corretta trascrizione dei suoni. Molto seria e motivata ad apprendere, appariva turbata dalla forte richiesta che lei esercitava su se stessa di rigore e precisione, sceglieva l'inglese per esprimersi e sembrava difficile convincerla ad abbandonarlo per utilizzare un italiano impreciso di cui si vergognava.

L'interazione più complessa è avvenuta con il bambino filippino che presentava varie difficoltà nell'articolazione fonatoria dei suoni e gran parte di ciò che diceva non risultava comprensibile. Sembrava convivere con uno stato di grande disagio e agitazione, esprimendo un comportamento molto vivace e faticando a portare a termine anche una semplicissima consegna con evidenti difficoltà a gestire i tempi e le modalità di lavoro. Si dimostrava comunque simpatico, curioso e disponibile alla relazione sia con i compagni che con l'insegnante.

33 La fase di analisi delle prove di ingresso ha orientato la programmazione delle attività didattiche e il reperimento di libri e materiali idonei al percorso. Sono stati dedicati anche ampi spazi alla progettazione di attività volte alla costituzione del gruppo di apprendimento e al mantenimento di un clima positivo e sereno che accompagnasse tutte le unità di apprendimento proposte dal laboratorio.

Dal terzo incontro il laboratorio è entrato nel vivo delle sue attività, con la presentazione dei contenuti delle unità di apprendimento.

La programmazione delle attività didattiche e dell'approccio metodologico scelto è partita dall'assunto che l'apprendimento di una lingua straniera, riferito a discenti della scuola primaria, rientra tra le competenze "semiotiche" nell'ambito espressivolinguistico del quale fanno parte, oltre all'educazione linguistica, anche l'educazione motoria, musicale e artistica (Balboni 2008). Sposare una logica di educazione semiotica ha richiesto l'assunzione di una prospettiva unitaria, rispettosa delle procedure globalistiche della mente del bambino e della suddivisione tra scopi pragmatici ed uso estetico che caratterizza ciascun linguaggio. 

laboratorio sono state quelle di essere:

- inclusiva tra le diverse educazioni dell'ambito linguistico espressivo articolando diverse attività didattiche dove l'input linguistico fosse connesso all'arte, alla motricità e alla musica,

- flessibile metodologicamente nel rispetto dei diversi stili e tempi di apprendimento

- fortemente ludica e sensoriale (Freddi 1990) l'acquisizione di una lingua straniera (Krashen 1985): la presentazione delle unità di apprendimento è stata in ordine di difficoltà crescente secondo l'ordine naturale di acquisizione, in continuità le une rispetto alle altre, considerando di volta in volta $i$ bisogni formativi espressi dagli alunni e adeguando a questi ultimi le proposte didattiche. quelli che ricevevano nella loro giornata scolastica, fosse un input comprensibile collocato nella loro zona di sviluppo prossimale, (Vygotsky 1980) e per ultimo, ma non meno importante in una situazione affettiva ed emotiva di sicurezza, protezione e accettazione, senza ansia di prestazione e paura di insuccessi.

A tale scopo, particolare attenzione è stata rivolta alla costruzione del gruppo di apprendimento affinché nell'approccio degli alunni vi fosse disponibilità ed empatia, all'inizio non presenti in tutti i componenti. Viceversa grande in tutti è stata fin da subito la motivazione rispetto all'apprendimento dell'italiano, dimostrando di gradire enormemente uno spazio e un tempo dedicato esclusivamente a quello, in cui poter chiedere e sbagliare sentendosi nel pieno diritto di farlo.

I bambini non sono stati considerati come "tabula rasa" ma si è riconosciuta loro una competenza plurima maturata nell'ambiente di vita prescolare nei loro paesi d'origine che emergeva continuamente in modo spontaneo o su richiesta come ad esempio nel momento iniziale "di riscaldamento" della lezione dove venivano chiesti dei termini nella L1 degli apprendenti riguardo ad argomenti che sarebbero stati oggetto di lezione, per sottolineare a loro stessi e agli altri la loro competenza nella lingua madre nel riconoscimento dell'eguale dignità e valore di tutte le lingue. L'italiano quindi come "lingua di contatto" (De Mauro 2000) definizione che fornisce agli insegnanti una chiave per progettare e un orientamento che segna tutto l'intervento di formazione linguistica, non dimenticando la complessità e la potenziale conflittualità della condizione dei suoi destinatari: bambini di famiglie immigrate in Italia.

41 Considerata l'urgenza comunicativa di questi bambini e osservando il loro disagio in diversi contesti di vita quotidiana a scuola, si è voluto promuovere al massimo lo sviluppo della competenza linguistico-comunicativa e in ciò centrale è stato il ruolo della comunicazione didattica che si è configurata su un modello "bidirezionale faccia a faccia con presa di parola libera" (Vedovelli 2002), offrendo la possibilità di avere moltissimi scambi verbali tra di loro e con l'insegnante, privilegiando l'oralità anche visto il limitato accesso alla scrittura (tre su quattro frequentavano la prima elementare) e il numero dei corsisti, aumentando così al massimo la densità comunicativa.

42 All'interno del corso non si è solo insegnata una lingua ma anche alcuni frammenti $d i$ cultura italiana che stanno "dentro" ad essa allo scopo di rendere sempre più efficaci i 
messaggi della loro vita quotidiana e con essi realizzare le funzioni principali della competenza socio-pragmatica (Balboni 2008).

Può essere interessante scendere più nello specifico in riferimento ad una delle attività didattiche presentate ai bambini per la sua significatività rispetto al contesto scolastico. Ne ripercorriamo brevemente il percorso.

Uno dei giochi presentati è stato "Il gioco dei comandi". La scrittura dei comandi e la costruzione del gioco è stata affidata ad una classe quinta dello stesso Istituto Comprensivo che, informata rispetto al laboratorio di italiano, è stata stimolata alla produzione di un'attività didattica per implementare l'apprendimento dell'italiano in modo giocoso. Questo gioco consiste nel dire un numero e prendere dal tabellone di cartone, aprendo una finestrella, il biglietto corrispondente, leggere la consegna ed eseguire l'azione indicata facendola indovinare ai compagni. Il compagno che indovina avrà la possibilità di dire un numero e pescare un nuovo bigliettino proseguendo il gioco. Vince chi esegue correttamente il maggior numero di comandi.

La partecipazione della comunità educativa nel suo complesso ad una piccola esperienza che l'Istituzione scolastica sta portando avanti è di particolare importanza rispetto alla relazione tra gli alunni nativi e stranieri. Tale compito vede la scuola primaria impegnata in prima fila rispetto alla formazione di un senso civico aperto, curioso e costruttivo rispetto alla convivenza tra italiani e figli di famiglie immigrate. Importante da questo punto di vista è poter contribuire attraverso una proposta concreta e saper fare qualcosa che veicoli un contenuto di vicinanza e aiuto rispetto al compito di apprendimento affidato a questi bambini. La consegna di tale oggetto è stata innanzi tutto un momento di conoscenza e di assunzione di responsabilità degli alunni più grandi della quinta classe rispetto ai piccoli e poi, in un secondo momento, anche un riconoscimento nel ruolo di tutor e ambasciatori della lingua italiana, creando una situazione di partecipazione e crescita per tutti.

Progettare uno spazio di apprendimento per accogliere, orientare e offrire i primi elementi nella L2 a questi alunni di recente immigrazione, in una scuola in cui l'esperienza del laboratorio di italiano non si era realizzata in precedenza, ha avuto una duplice ricaduta positiva. Da una parte ha rafforzato le competenze nella lingua italiana di questi alunni. Dall'altra ha aumentato la visibilità degli specifici bisogni nella formazione linguistica di questi bambini, rendendo anche la comunità scolastica più consapevole della necessaria presa in carico e responsabilità che essa deve avere verso le difficoltà comunicative emerse.

Questo ascolto e questa attenzione ricevuti nella prima delicata fase di inserimento scolastico ci auguriamo possa costituire una premessa positiva su cui si fonderà la personalità bilingue (Freddi 1988) che andranno a sviluppare, sia da un punto di vista linguistico che sociale. Per adesso ciò che possiamo registrare in base ai risultati dei test somministrati e delle considerazioni emerse da parte delle insegnanti che hanno nelle loro classi questi alunni, è che essi hanno sviluppato maggiore fiducia in se stessi e sicurezza nelle proprie abilità di neoparlanti dell'italiano, in modo da rafforzare così il senso di autoefficacia in una realtà vissuta all'inizio con timidezza e diffidenza.

Pensiamo che il laboratorio abbia sostenuto e affiancato gli alunni nel loro processo di educazione linguistica (per la maggior parte di loro anche in concomitanza con il processo di apprendimento della letto-scrittura stessa) contribuendo a creare le condizioni per un inserimento più sereno e positivo nella nuova realtà di riferimento. Grande importanza hanno avuto il riconoscimento del ruolo genitoriale e il rapporto 
collaborativo con le famiglie di questi alunni così come il rispetto dei tempi di apprendimento ed inserimento dei bambini. Con il fine ultimo di aggiungere questa al ricco patrimonio delle esperienze che vogliono contribuire all' integrazione nel sistema scolastico e sociale di alunni stranieri immigrati.

Sono ormai molti anni che la scuola primaria si confronta con la presenza di alunni stranieri nelle classi e fortemente è sentita la necessità di supportare il loro apprendimento linguistico affinché questi alunni non confluiscano all'interno di quella fascia debole segnata da fallimento, difficoltà, ritardi e abbandono scolastico di cui $\mathrm{i}$ dati prima presentati ci parlano chiaramente.

Di fronte a questa sfida educativa da cui la scuola tutta potrebbe trarre grande giovamento, prendono vita iniziative di vario genere volte a potenziare la conoscenza dell'italiano e la comprensione profonda della lingua scritta e orale per consentire ai minori stranieri la maggior competenza scolastica e sociale possibile in vista di una loro futura cittadinanza italiana.

\section{BIBLIOGRAFIA}

BALBONI P. 2002. Le sfide di Babele. Insegnare le lingue nelle società complesse. Torino, UTET.

BALBONI P. 2008. Imparare le lingue straniere. Venezia, Marsilio.

DE MAURO T. 2000. Commissione ministeriale sui curricoli della scuola di base. MIUR.

FREDDI G. 1988. Lingue straniere e istruzione primaria in Italia e in Europa. Padova, Liviana

Scolastica.

FREDDI G.1990. Il bambino e la lingua. Psicolinguistica e glottodidattica. Padova, Liviana.

KRASHEN S. 1985. Second Language Acquisition and Second Language Learning. Oxford, Pergamon Press,

VYGOTSKY L. 1980. Il processo cognitivo, Torino, Boringhieri.

VEDOVELLI M. 2002. Guida all'italiano per stranieri. La prospettiva del quadro comune europeo per le lingue, Roma, Carocci.

\section{NOTE}

1. Si coglie qui l'occasione per ringraziare la Dirigente scolastica, gli insegnanti, le famiglie e i bambini che hanno collaborato e reso possibile questa esperienza. 


\section{RIASSUNTI}

L'expérience didactique décrite ici s'est déroulée pendant l'année scolaire 2017-2018 dans une école primaire de l'Istituto Comprensivo en périphérie nord-est de Rome. Avant de donner les détails de l'expérimentation, nous présentons un aperçu des statistiques concernant les élèves étrangers en Italie, qui permettent une meilleure compréhension du phénomène. Par rapport à l'expérience pédagogique réalisée, nous examinerons les méthodes, les modules d'apprentissage, les phases du curriculum et d'autres facteurs qui peuvent donner au lecteur une idée de la manière dont le laboratoire de langues fonctionne.

The teaching experience described here took place during the 2017-2018 school year, in an elementary school of the Istituto Comprensivo at the North-Eastern periphery of Rome. Before going into the details of the experiment, we will present a review of the statistical data concerning the foreign pupils in Italy, which provides some significant information for a better understanding of the phenomenon. Corresponding to the pedagogical experience at hand, we shall examine the methods, the learning module, the phases of the curriculum and other factors that allow the reader to see how the language laboratory functions.

\section{INDICE}

Keywords : Italian as a second language, newly arrived foreign pupils, elementary school, learning through games, languages in contact

Mots-clés : Italien L2, élèves étrangers primo-arrivants, apprendre par le jeu, langues en contact.

\section{AUTORE}

\section{FEDERICA GAETANO}

Scuola primaria I C Nicolai, Roma (Italie) 